\title{
Strong Interactions of Single Atoms and Photons in Cavity QED
}

\author{
H. J. Kimble* \\ Norman Bridge Laboratory of Physics 12-33, California Institute of Technology, Pasadena, CA 91125, USA
}

Received September 19, 1997; revised version received January 1, 1998; accepted January 14, 1998

PACS Ref:

\begin{abstract}
An important development in modern physics is the emerging capability for investigations of dynamical processes for open quantum systems in a regime of strong coupling for which individual quanta play a decisive role. Of particular significance in this context is research in cavity quantum electrodynamics which explores quantum dynamical processes for individual atoms strongly coupled to the electromagnetic field of a resonator. An overview of the research activities in the Quantum Optics Group at Caltech is presented with an emphasis on strong coupling in cavity QED which enables exploration of a new regime of nonlinear optics with single atoms and photons.
\end{abstract}

\section{Introduction}

One of the most important themes of quantum mechanics since its inception has been quantum measurement. Beginning with the then startling revelation of the impossibility of noninvasively monitoring a quantum system as codified by the Heisenberg uncertainty relations, advances in our understanding of the "rules and regulations" governing the flow of information to and from quantum systems have had a profound impact on the epistimology of science. As anticipated in the seminal work of Einstein, Podolsky, and Rosen [1], a landmark achievement in this endeavor was provided by John Bell, who changed once and for all our understanding of locality and realism in the quantum world [2].

Bell's work ushered in a new era in quantum measurement driven by experimental investigations [3]. Prior to this time and indeed throughout most of its history, the subject of quantum measurement had all too often been a metaphysical enterprise due to a persistent fascination of theory with questions of no operational significance and to a lack of technical tools sufficient to the task of laboratory investigations. However, over the past three decades this situation has changed profoundly with major advances in a number of areas and with a level of activity that shows no signs of abatement, as evidenced by the presentations at this Symposium. Rather than attempt to trace all of the threads that have been woven to make the fine tapestry that has emerged, here I wish to concentrate on the origins of revolution sown in two particular research communities.

Beginning in the somewhat unlikely quarter of attempts to detect a classical force, Vladimir Braginsky, Kip Thorne, and their colleagues (who were investigating the possibility of the detection of gravitational radiation) recognized the relevance of quantum mechanics as applied to a macroscopic mass in limiting the resolution of a "bar antenna" [4-6]. Driven by their vision of a new window on the universe via gravitational-wave astronomy (and at least not initially by a love of quantum measurement per se), these explorers for-

*email: hjkimble@cco.caltech.edu mulated but were not daunted by the standard quantum limit $(S Q L)$ which delineated the then known boundary of knowledge. Instead they pressed forward to invent new measurement strategies, including most significantly the concept of quantum non-demolition measurement $(Q N D)$, whereby the $S Q L$ could be circumvented.

A second community engaged in a seemingly disjoint journey was that of quantum optics, who since the pioneering days of Glauber [7] and Sudarshan [8] in the 1960s had been pursuing their own "golden fleece" in the form of states of the electromagnetic field with manifestly quantum or nonclassical character. Although surely the correct description of the electromagnetic field is a quantum one, just as surely the vast majority of optical phenomena are equally well described by a semiclassical theory, with atoms quantized but with a classical field. In this regard, Einstein is purported to have said, "I know that beer comes in pint bottles" (in refering to the quantum features of blackbody radiation). "What I want to know is whether all beer comes in pint bottles" (that is, whether the 'quantum character' of the electromagnetic field has its origins in the atoms that bring the field to thermodynamic equilibrium or as an intrinsic property of the field). The first experimental example of a manifestly quantum or nonclassical field was provided in 1977 with observations of photon antibunching for the fluorescent light from a single atom [9].

As is often the case in science, many profound discoveries occur in the unexplored domains between more established territories. In the story at hand, Carleton Caves played a key role in bridging the gap between the aforementioned gravitional-wave and quantum optics communities, and catalyzed especially the later to action. In various disguises, Caves and others [10] demonstrated that zero-point or vacuum fluctuations were the villians responsible for the SQL and that they could be vanquished by the nonclassical states of quantum optics [11]. Nonclassical states thus became essential ingredients in advancing measurement science beyond the SQL. After initial work by Slusher and Yurke in 1985 to generate squeezed states of light [12], the first measurement with sensitivity beyond the SQL was reported [13].

There has since ensued an expanding zoology of nonclassical states of the electromagnetic field [14], with diverse "proof-of-principle" demonstrations ranging from spectroscopy $[15,16]$ to communication $[17,18]$ to the realization of the original EPR gedanken experiment [19]. Although these experiments have been conceptually important, a common ingredient to them all has been the lack of a truly spectacular advance beyond the borders delineated by the SQL, with factors of two rather than ten-to-the-two characterizing the state of the art [20]. Even in light of the uncompromising task master which is the standard quantum limit, 
this situation must be viewed as somewhat disappointing given that we are now twenty years past the debut of nonclassical light.

An inquiry into the root cause of this state of affairs can easily become mired in the technical details of any one system, which nonetheless surely does lead to ideas for further advances. However, it is my view that truly spectacular progress is blocked in more generic terms by our inability to make an operational accounting quantum by quantum for system losses, which in turn is related to the ratio of times scales for coherent, reversible evolution to that for irreversible, dissipative loss for the systems employed. In this regard, it is important to note that although the first demonstrations have been made for the electromagnetic field within the realm of quantum optics, quantum measurement has advanced on other fronts as well. A notable but certainly not exhaustive list includes recent observations of squeezing for phonons [21] and of the quantum statistical character of electron transport [22]. Here too, the effects reported have been modest.

Leaving aside for the moment this tale of the trials and tribulations of quantum measurement, let us turn to another important trend of modern physics, namely an unrelenting march to isolate and manipulate the dynamical processes of individual quantum systems, with interactions studied quantum by quantum. In optical physics, one example of such research is cavity quantum electrodynamics with single atoms and photons, while in condensed matter physics, a notable example is Coulomb blockade with discrete electron energies [23]. Note that the emphasis is not simply to observe event by event as in traditional scattering experiments, but rather to control quantum evolution in a deterministic fashion, where the sense of this seemingly contradictory phrase will be made clear shortly.

Just as in the discussion of quantum measurement where the convergence of disparate communities made possible a series of spectacular advances, another potentially profound confluence is underway with the merging of quantum information science with the ability for quantum control of the dynamics of complex systems. Examples in this arena include quantum computation and communication, which require for their implementation the ability to manipulate quantum systems in a prescribed fashion at the level of individual constituents (be they photons, phonons, or electrons) [24]. Within this context, I would like to advance the point of view that quantum measurement should now rightly be considered as subsumed within the broader context of quantum information science. Indeed, it is my belief that quantum measurement will not pass beyond its current plateau of proof-of-principle demonstrations until and unless we learn how to implement diverse quantum control algorithms, including quantum error correction to conquer the debilitating effects of dissipation for open quantum systems.

An essential ingredient in this endeavor is the ability to bring two components of a complex quantum system together in a control fashion. Qualitatively, assume that the off-diagonal components of the system's interaction Hamiltonian are characterized by $\left\langle H_{\text {int }}\right\rangle \sim \hbar \chi$, where $\chi$ is the rate of coherent, reversible evolution [25]. If the interaction proceeds for time $T$ (which must be under our external control), then a necessary requirement for conditional quantum dynamics whereby one component has appreciable impact on a second component of the system is for the quantity $\theta=\chi T \sim 1$. Certainly, in any real world situation, there will be irreversible interactions of the system with the external environment, which lead to dissipative decay at rate $\Gamma$ for any individual constituent (e.g., decay of a quantum spin). In addition to the requirement $\theta \sim 1$, we must also then have $\chi \gg \Gamma$.

In terms of these criteria for $\chi$, it is straightforward to divide the future from the past by noting that almost without exception, previous experimental investigations in the domain of quantum measurement and information science have been carried out in a regime of weak coupling, for which $\chi \ll\left(T^{-1}, \Gamma\right)$. By contrast, the future of this field depends upon the ability to operate in a domain of strong coupling, for which $\chi>T^{-1} \gg \Gamma$. Note that the quantity $m_{0}=\Gamma^{2} / \chi^{2}$ plays the role of a "critical" number of quanta for such a system, where with few exceptions in physics, $m_{0} \gg 1$ is the rule so that single quanta have negligible impact on the system's dynamics.

The Nobel Symposium has provided beautiful examples to illustrate the broad front on which research is being pursued into the largely unexplored domain of strong coupling, with the work of M. Devoret (see contribution by Bouchiat et al.) being a notable example from the condensed matter community and that of $\mathrm{D}$. Wineland from the area of cooling and trapping of individual ions in AOM physics. A third area is that of cavity QED, with exemplary presentations by $\mathrm{S}$. Haroche and $\mathrm{H}$. Walther describing their research in the microwave domain. The Quantum Optics Group at Caltech pursues research in the area of cavity QED in the optical domain. Although there are many facets to our endeavor, the primary motivation is the quest to exploit strong coupling in cavity QED as an enabling capability for research in quantum measurement and more generally, in the emerging field of quantum information dynamics. It is to this cavity $Q E D$ circus with flying photons, falling qubits, and fantastic finesse that I would like now to focus attention.

\section{Cavity quantum electrodynamics}

As illustrated in Fig. 1, our model system is taken to consist of a single two-state atom located in an optical cavity formed by two spherical mirrors. The Hamiltonian $H_{\mathrm{s}}$ for

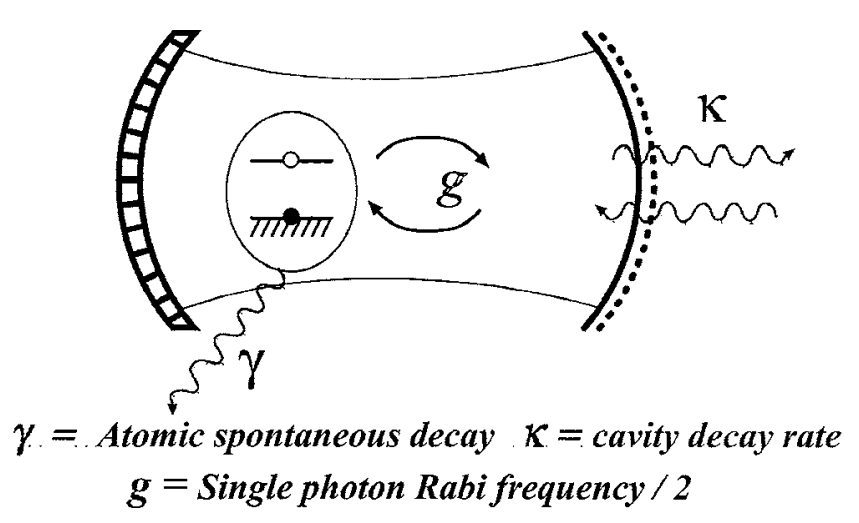

Fig. 1. Schematic illustrating a two-state atom interacting with the quantized field of an optical cavity with coupling coefficient $g$. In addition to this reversible evolution are irreversible decay channels denoted by $(\gamma, \kappa)$. 
this system is well-known and takes the form of a sum of atomic, field, and interaction terms [26-28],

$\hat{H}_{\mathrm{s}}=\frac{\hbar \omega_{\mathrm{A}}}{2} \hat{\sigma}^{z}+\hbar \omega_{\mathrm{C}} \hat{a}^{\dagger} \hat{a}+\mathrm{i} \hbar\left[g(\boldsymbol{r}) \hat{a}^{\dagger} \hat{\sigma}^{-}-g^{*}(\boldsymbol{r}) \hat{a} \hat{\sigma}^{+}\right]$.

The operators $\hat{a}$ and $\hat{a}^{\dagger}$ are the annihilation and creation operators for the single-mode of the resonator under consideration, while $\hat{\sigma}^{z}$ and $\hat{\sigma}^{ \pm}$are the Pauli operators for the atomic inversion, raising, and lowering, respectively. $\left(\omega_{\mathrm{A}}\right.$, $\left.\omega_{\mathrm{C}}\right)$ are the atomic and cavity resonance frequencies. The coherent coupling between the atom at position $\boldsymbol{r}$ and the cavity mode is $g(\boldsymbol{r})$, with

$g(\boldsymbol{r})=\left(\frac{\mu^{2} \omega_{\mathrm{C}}}{2 \hbar \varepsilon_{0} V_{m}}\right)^{1 / 2} U(\boldsymbol{r}) \equiv g_{0} U(\boldsymbol{r})$,

where the cavity-mode function $U(\boldsymbol{r})$ is chosen so that the cavity-mode volume $V_{m}=\int \mathrm{d}^{3} x|U(\boldsymbol{r})|^{2} . \mu$ is the transitiondipole moment for the (assumed) two-state atom. Of course $\hat{H}_{\mathrm{s}}$ is responsible for coherent (reversible) evolution in our problem, with $g_{0}$ playing the role of $\chi$ from the previous section.

In addition to the dynamical processes associated with $\hat{H}_{\mathrm{s}}$ alone, we must also include the ubiquitous dissipative processes arising from coupling of the atom to modes of the electromagnetic field other the privilidged mode of the resonator. By way of a weak-coupling approximation for the atomic interaction with these "external" modes [28], we arrive at rates $\left(\gamma_{\|}^{l}, \gamma_{\perp}^{l}\right)$ for longitudinal and transverse decay of the atomic energy and polarization. While these rates are in general specific to the location $\boldsymbol{r}_{l}$ of the atom within the cavity (as is the position dependent radiative frequency (Lamb) shift $\delta^{l}$ ) [29], it suffices in many cases to neglect variations in $\left(\gamma_{\|}, \gamma_{\perp}\right)$ with position $\boldsymbol{r}_{l}$ [and also in $\delta$ ], although we have recently analyzed the radiative coupling of an atom to the whispering gallery modes of quartz microspheres where this is not the case [30]. Damping of the cavity mode through the boundaries of the resonator is likewise accounted for by a rate $\kappa$, where now $(\gamma, \kappa)$ play the role of $\Gamma$ from the preceding section.

Even with this brief statement of the problem, considerable insight into the nature of the dynamical processes can be obtained by introducing two dimensionless parameters formed from the three rates $\left(g_{0}, \gamma, \kappa\right)$ [Here we take $\gamma \equiv$ $\gamma_{\perp}=\gamma_{\|} / 2$ as appropriate to radiatively broadened decay to external background modes]. We define

$n_{0} \equiv\left(\frac{\gamma_{\perp} \gamma_{\|}}{4 g_{0}^{2}}\right) b=\frac{4}{3} \frac{\gamma^{2}}{g_{0}^{2}}, \quad N_{0} \equiv 2 \gamma_{\perp} \kappa / g_{0}^{2}=2 \gamma \kappa / g_{0}^{2}$,

where $b=\frac{8}{3}$ for an average over a Gaussian standing-wave mode, while $b=1$ for an atom with $U(\boldsymbol{r})=1$ [25]. Note that $n_{0}$ gives the "saturation" photon number for the atomcavity field interaction, while $N_{0}$ serves as a measure of the "critical" atomic number $\left(N_{0}^{-1}\right.$ gives the cooperativity parameter per atom) [27]. In qualitative terms, $\left(n_{0}, N_{0}\right)$ specify the role of a single photon and of a single atom, respectively.

From its inception in the 1960 s, quantum optics has been a field concerned almost exclusively with processes in a regime of weak coupling for which $g_{0} \ll(\gamma, \kappa)$, so that $\left(n_{0}\right.$, $\left.N_{0}\right) \gg 1$, and for which one photon or one atom more or less is of no consequence. For example, note that a typical laser is operated with a threshold photon number $\sqrt{n_{0}} \sim$ $10^{3}-10^{4}$ and that typical nonlinear optical processes such as parametric down conversion have $\sqrt{n_{0}} \gtrsim 10^{4}-10^{5}$ photons, with the number of atoms $N_{0} \gg 1$ in each case. In this limit of weak coupling, the quantum master equation can be solved by a system-size expansion based upon the small parameters $\left(n_{0}^{-1}, N_{0}^{-1}\right)[20,28]$, with the generic result that dynamical processes take the form of more or less classical trajectories with small bits of quantum noise. Note that in this case, the internal time scale $g_{0}^{-1}$ for coherent quantum dynamics is scaled away.

This is not to say that processes in a domain of weak coupling are without interest from the perspective of quantum measurement and information science. Indeed, the first measurements to generate and apply nonclassical states to such problems (as well as many of the contributions to the Nobel Symposium) were carried out in a domain of weak coupling. However, as described in more detail in Ref. [31], weakly coupled systems are not capable of providing $a$ priori quantum states of the field in a controlled fashion, but rather offer only a posteriori post dictions of such behavior, as for example, in experiments to explore entanglement via photon pairs generated via parametric down conversion, including recent work on teleportation $[32,33]$.

By contrast, in the regime of strong coupling the internal clock which specifies coherent quantum time $g_{0}^{-1}$ runs faster than the external dissipative clock $\left(\gamma^{-1}, \kappa^{-1}\right)$. The atomcavity system then has time to couple itself coherently and at least the possibility of a life of manifestly quantum dynamics before the grim reaper of dissipation enters. It is to this domain of strong coupling that we turn our attention in a quest to explore qualitatively new phenomena in quantum optics associated with the exquisite interplay of coherent and dissipative dynamics.

While it is straightforward to specify the criteria necessary for strong coupling (i.e., $g_{0}>(\gamma, \kappa)$ ), it is an altogether different task to achieve these conditions in the laboratory, as evidenced by the paucity of realizations across physics generally. Our progress in this regard is illustrated in Fig. 2, which plots the critical photon number $n_{0}$ achieved for a series of our experiments dating to 1979 when our effort began. Note that most recently, we have implemented a system with a cavity of length $10 \mu \mathrm{m}$ and mirrors with radii $R=5 \mathrm{~cm}$, for which

$\left(g_{0}, \gamma, \kappa\right) / 2 \pi=(120,2.5,35) \mathrm{MHz}$

and

$\left(n_{0}, N_{0}\right)=\left(2 \times 10^{-4}, 1.2 \times 10^{-2}\right)$,

which is the last point in Fig. 2 [34]. An essential underlying technical advance that has enabled the steady reduction of $n_{0}$ evidence in Fig. 2 has been an ever increasing rise in the finesse for the spherical mirror interferometers employed in our research, beginning with finesse values $\mathscr{I} \sim 10^{2}$ in 1980 and reaching $\mathscr{I}=1.9 \times 10^{6}$ in 1992 (i.e., mirror reflectivity $R=0.9999984$ and cavity $Q=1.8 \times 10^{10}$ (for length $l=4 \mathrm{~mm}$ )) [35], which remained for many years the highest finesse recorded for an optical cavity. However, recently we have pushed to somewhat a higher value $\mathscr{I}=2.2 \times 10^{6}$ for the whispering gallery modes of quartz microspheres [36]. 


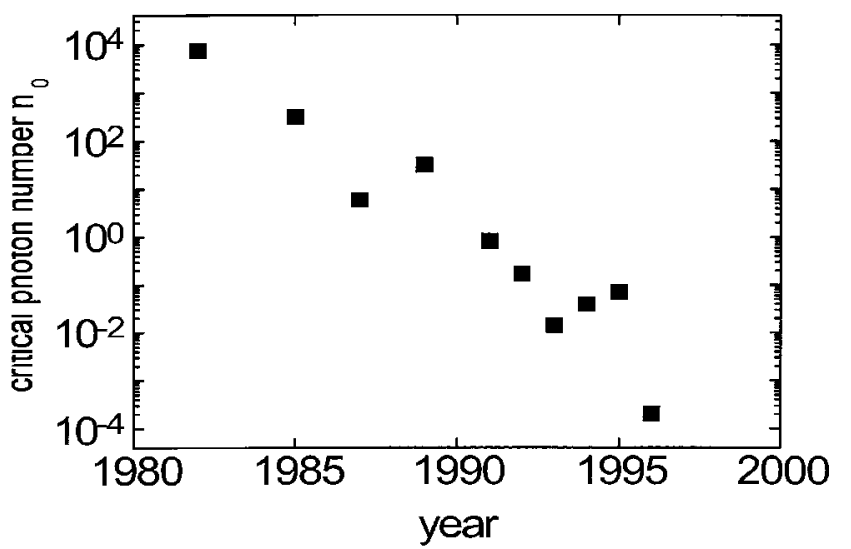

Fig. 2. Critical photon number $n_{0}$ vs. year. The data for this plot are described in Ref. [25], with the exception of the last two points which are from Refs [34, 48], respectively.

Within the context of other experimental work in cavity QED, there are two experiments in the microwave domain which employ Rydberg atoms in high- $Q$ superconducting cavities and which are reviewed by the contributions of $S$. Haroche [37] and H. Walther [38] in these proceedings. By following our lead, the group of M. Feld has also achieved strong coupling in the optical domain [39].

\section{Survey of research activities}

In the following sections, we briefly review some of our scientific activities enabled via strong coupling in cavity QED. The discussion is a blend of advances to date together with glimpses of future prospects to whet the appetite, with a common theme being a continuation of the quest for lowloss resonators of small mode volume. In addition, the marriage of laser cooling and trapping with cavity QED should lead to a splendid set of progeny from the interplay of internal (atomic dipole + quantized cavity field) and external (atomic center-of-mass wavepacket) degrees of freedom.

We will not attempt to describe our past work in cavity QED, for which a review is available in Ref. [25], choosing to focus instead on current and future activities. Suffice it to say that an important component has been an investigation of "structural" issues to validate the shared partnership of field and atom, and has included observations of the "vacuum-Rabi" splitting for a single atom [40, 41] and of the nonlinear response of the system [41, 42]. Here "structure" refers to the eigenstates and eigenvalues which follow from the diagonalization of the system Hamiltonian $H_{\mathrm{s}}$ [43]. In addition to this work on "spectroscopy" of the atom-cavity system, we have also studied quantum dynamical processes in a regime of strong coupling, such as quantum-state reduction leading to photon antibunching [44]. This work has provided an explicit demonstration of the failure of traditional system size expansions in describing open quantum systems in a domain of strong coupling. Note that the atom-cavity system is a particularly attractive system for these investigations because of an absence of complicating nonessential features; both the reversible atom-field interaction and the irreversible dissipative processes are fundamental radiative interactions that are well understood as constituent pieces. Complexity arises from the interplay of these otherwise fundamentally simple, wellcharacterized components.

\section{Nonlinear optics with single photons and atoms}

As described in Ref. [45], by suitably coupling an atom to a single mode of a high-finesse cavity, it is possible to create an effectively "one-dimensional" atom, whereby the dominant channel for atomic radiative interactions is via the cavity mode and thence to and from the external environment. In this setting, input fields such as squeezed light and monophotonic pulses can be generated for excitation of the atom-cavity system, and output fields can be detected with high efficiency by various strategies. An example of the nonlinear response of such a "one-dimensional" atom is shown in Fig. 3, which plots the normalized transmission of the atom-cavity system $v s$. the mean intracavity photon number $m$. As previously discussed, note that a single intracavity atom has a large impact on the cavity properties (for $m \rightarrow 0$, the transmission is reduced to $20 \%$ of its value for the empty cavity with no atoms, illustrating the role of $N_{0}$ ). More recent measurements record a reduction of $10^{-2}$ for the passage of a single atom [34]. Likewise, "saturation" onsets for $m \approx 0.02$ intracavity photons (on average), in correspondence to the critical photon number $n_{0}=0.02$ for this system. Current research centers on a system with $n_{0}=2$ $\times 10^{-4}$.

One application of these capabilities for nonlinear optical processes with $n_{0} \ll 1$ photons is to the implementation of quantum logic in cavity QED. Indeed, we have made the first demonstration of conditional dynamics at the single photon suitable for this purpose, which we termed a quantum-phase gate (QPG) [46, 47]. Our measurements utilized the circular dichroism of an atom strongly coupled to the field of a high finesse optical cavity to rotate the polarization state of a linearly polarized "probe" beam (i.e., a one-atom waveplate). Because the rotation angle of the probe beam could be controlled by the intensity of a circularly polarized "pump" beam for intracavity fields with average photon number much less than one, our observations demonstrated conditional dynamics between pump and probe fields at the level of single quanta (the analog of the Kerr effect, here for single quanta). The "truth table" for our quantum-phase gate was experimentally verified, with

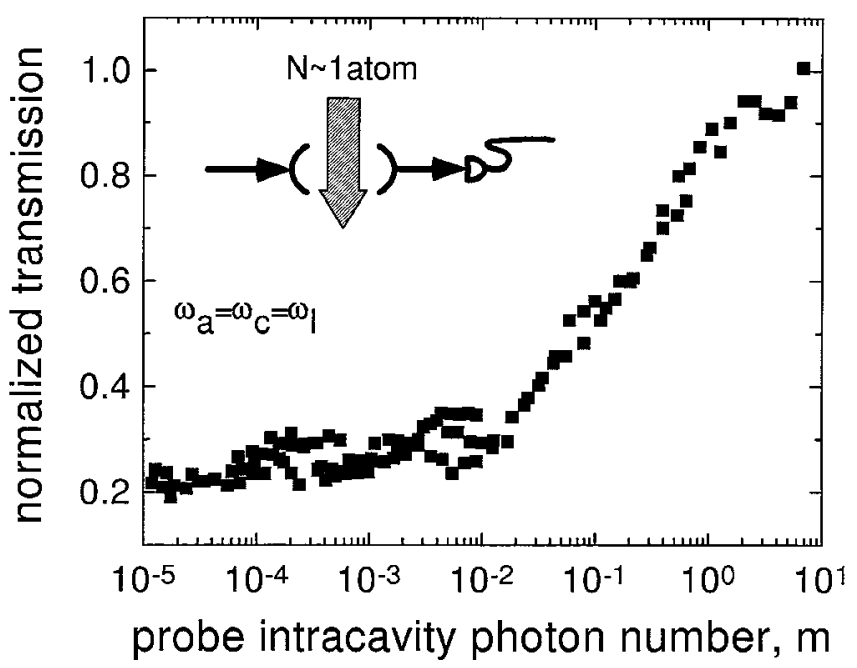

Fig. 3. Transmission of the atom-cavity system vs. mean intracavity photon number $m$. Note the onset of a nonlinear response for $m \approx 10^{-2}$ photons. These measurements are for a probe field resonant with the coincident atom and cavity frequencies. The transmission is normalized to that of the empty cavity (no atoms). (Ref. [46]). 
the measurements indicating that the transformation affected by the atom-cavity system is "non-trivial" in that it could serve as a universal element for quantum computation. Here the quantum carriers of information (the "qubits") are fields which propagate in two frequency offset (and hence functionally distinct) channels, with the internal state in each case specified by the circular polarization states $\sigma_{ \pm}$. Although we have not made measurements of entanglement for the fields emerging from the QPG, detailed calculations carried out by $\mathrm{S}$. Tan (University of Auckland) indicate that it should be possible to observe nearly maximal violations of Bell inequalities for the output fields (even with coherent-state inputs). Note that beyond the context of quantum logic, the large values recorded for the dispersive nonlinear interaction between intracavity fields represents a unique achievement within the field of nonlinear optics.

\section{Real-time cavity QED with individual atoms}

A major technical shortcoming of our work in cavity QED has been that atomic beams have been employed, so that there are unavoidable fluctuations in atomic number and position. To remedy this situation, we have undertaken a program to localize single atoms within the cavity at welldefined positions. The first step in this process is the realtime detection of single atoms transiting through a high-finesse optical cavity [34, 48]. For these experiments, Cesium atoms are dropped from a magneto-optical trap (MOT) located a few millimeters above a Fabry-Perot cavity, as illustrated in Fig. 4. By recording the reduction of the cavity transmission as an atom enters the cavity mode, we can monitor the "trajectory" of an individual atom as it transits through the cavity, with detailed examples of such data given in Ref. [34, 48] and with changes in cavity transmission of $10^{2}$ having been recorded for the transit of a single atom as indicated in the figure [34].

Some sense of the advance that this work represents relative to other experiments in the area of cavity QED is obtained by noting that the product of coherent coupling constant $g$ with the transit time $T$ is $g_{0} T \sim \pi \cdot 10^{3}-10^{4}$, whereas for all other experiments (which employ atomic beams), $g_{0} T \sim \pi[25,37-39]$. Because the "optical information" per atom is given by $I \equiv\left(g_{0}^{2} / \kappa\right) T$ and $I \gg 1$ in our experiments, we are thus able to extract detailed information about the atom-field dynamics for a single atom in real time. For example, we have recently made observations of the "vacuum-Rabi" splitting based upon the system response atom-by-atom [34]. Likewise, we have made measurements of nonlinear optical response in the domain $m \ll 1$ intracavity photons (on average) as in the preceding section, but now recorded for individual atomic trajectories [34]. By contrast, note that for experiments in the microwave domain, the transit of a single atom leads to one bit of information (i.e. the atom is measured to be in either the ground or excited state), with the transit of many atoms required to obtain a meaningful measurement [37, 38]. Beyond the domain of cavity QED, the work reported in Ref. [48] represents an improvement of $10^{5}$ over previous work aimed at detection of single atoms or molecules by absorption [49, 50]. Our more recent activities as described
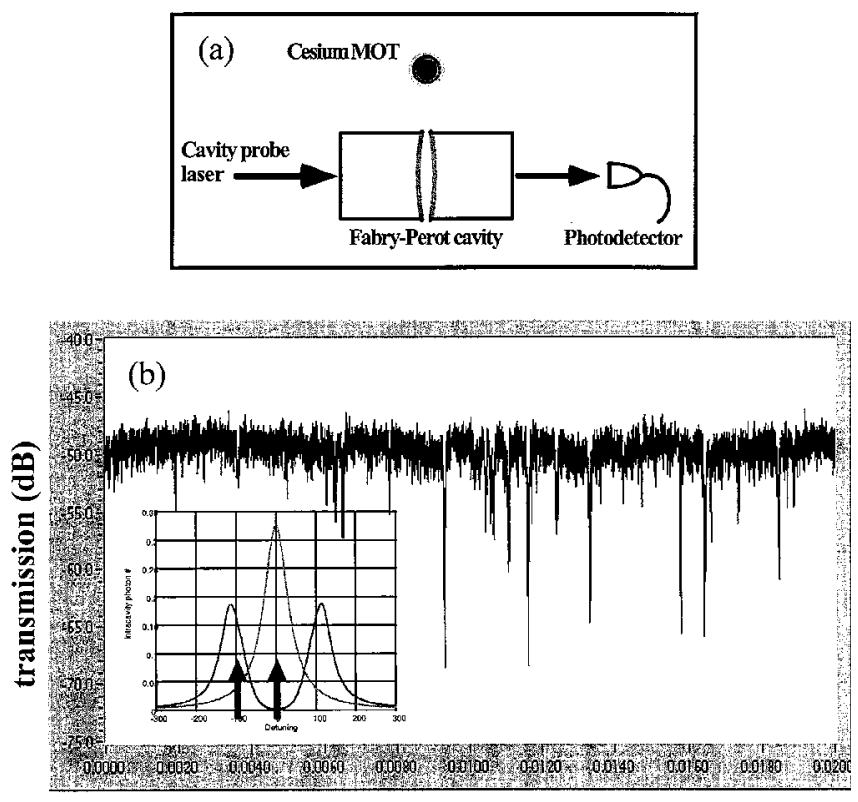

time (s)

Fig. 4. Schematic diagram of our experiment with cold atoms. (b) Cavity transmission vs. time after dropping the Cesium atoms from the MOT. The sharp down going spikes correspond to the transits of individual Cesium atoms, are of duration $\sim 100 \mu \mathrm{s}$, and represent atomic detection near the quantum limits. Note that the cavity transmission is given on a logarithm scale, with $m \approx 1$ photon as the steady-state level (at $-50 \mathrm{~dB})$ and with reductions approaching $10^{2}$ for some atom transits through the center of the cavity mode. The inset illustrates the empty cavity profile (the central Lorentzian) and that in the presence of an atom (the "vacuum-Rabi" splitting) for coincident atomic and cavity frequencies. These data are taken for resonant excitation (the central arrow in the inset), while to trap an atom, we switch during a transit to drive the lower component of the vacuum-Rabi doublet as indicated by the second arrow on the left. See Ref. [34].

in Ref. [34] demonstrate so-called "interaction-free" measurements at the level of single atoms and photons [51], and lay the foundation for the demonstration of a quantum switch [52], which can be in a coherent superposition of "open" and "closed".

\section{Continuous quantum measurement and the SQL}

Of course our ability to monitor atomic trajectories by the schemes discussed above cannot improve indefinitely, since at some point we must confront the quantum limits for detection of atomic motion. Stated more formally, we must move from a semiclassical description of the atomic centerof-mass motion to a fully quantum description of the atomic wavepacket, including the impact of the measurement process on the system's dynamics. Here the context of the research is that of the dynamics of continuously monitored quantum systems whereby the strong coupling of atom and cavity implies a back reaction of one subsystem on the other as a result of a measurement [53-55]. As applied to measurements of the atomic CM motion, we are particularly interested in the ultimate limits with which the atomic trajectory can be followed.

The basic mechanism for sensing atomic position within the cavity mode is through the spatial dependence of the cavity mode-function $g(\boldsymbol{r})$. In the case of a constant external 
drive, the magnitude and phase of the intracavity field $x$ depend in a self-consistent fashion on the atomic position $\boldsymbol{r}$. By monitoring the transmitted and reflected fields from the cavity with high bandwidth and efficiency, we gain information about the atomic position, albeit with a concomitant (measurement-induced) back-reaction on the atomic motion. Stated more explicitly, as the external drive and thereby the intracavity field increases, our "sensing" error (e.g., due to fluctuations in photon number) for estimating the intracavity field decreases, with correspondingly better resolution for the atomic position $\boldsymbol{r}$. On the other hand, an increase in the intracavity field perturbs the atomic motion (which is then no longer "free"), so that as the "sensing" error decreases, the "back-reaction" from the measurement increases. The standard quantum limit (SQL) for sensing atomic position is reached when the "sensing" and "backreaction" contributions to the uncertainty of atomic position are equal. For the case of impulsive measurements separated by a time interval $\tau$, the SQL for the position of a free particle of mass $m$ is given by the well-known expression [5]

$\Delta z_{\text {sql }}=\sqrt{\hbar \tau / m}$.

The SQL as expressed by this equation applies to certain "in principle" situations. However, the extension of this limit to more practical settings such as our work in cavity QED is not completely straightforward. For example, the inference of the atomic coupling $g(\boldsymbol{r})$ (and thence the atomic position $\boldsymbol{r}$ ) from measurements of the field external to the cavity is itself a nontrivial problem within the context of quantum parameter estimation (QPE) for a dynamical quantum system, as has recently been analyzed by $\mathrm{H}$. Mabuchi of our group [56]. Although considerable progress has been made on the general front of continuous position measurements for open quantum systems [53-55], we believe that better defined criteria are necessary in order to frame more clearly the operational signatures and significance of the SQL.

These comments notwithstanding, some indication that we are nearing an interesting frontier with respect to quantum position measurements is provided by an explicit evaluation of eq. (5) for our experiments with cold atoms $[34,48]$. For atom-transit signals as in Fig. 3 of Ref. [48], we observe a broad envelope which we associate with the Gaussian variation of $g(\boldsymbol{r})$ as the atoms fall along the vertical direction. However, many of the signals display additional, highly structured variations within this overall envelope with a typical "period" of roughly $\tau \sim 30 \mu \mathrm{s}$. Given initial transverse velocities $v_{\mathrm{t}} \sim \pm 1.5 \mathrm{~cm} / \mathrm{s}$ along the cavity axis (as set by the collimation provided by the mirror substrates), we then infer a distance scale $\delta z \sim 400-500 \mathrm{~nm}$ associated with this structure, which corresponds quite nicely with that expected for the standing-wave structure of the cavity field with periodicity $\lambda / 2=426 \mathrm{~nm}$. We have thus tentatively assigned this structure to atomic motion through the sinusoidual variation in $g(\boldsymbol{r})$, with the principal caveats being the role of recoil-induced heating and our limited detection bandwidth. On the other hand, if we evaluate eq. (5) for $\tau \sim 30 \mu \mathrm{s}$, we find $\Delta z_{\text {sql }} \sim 100 \mathrm{~nm}$, so that $\delta z \sim 4 \Delta z_{\text {sql }}$. Thus these initial measurements are already remarkable close to the naive quantum limit for sensing atomic position, although we are well aware that the evidence to date is suggestive and non conclusive.
Against this backdrop, our current experimental efforts are being devoted to making more definitive observations to connect the recorded variations in transmitted intensity to atomic motion through the standing waves, including recent measurements of the time evolution of the phase as well as of the amplitude of the intracavity field during an atomic transit for nonzero detunings. Within a broader context, we are attempting to explore the issue of "realism" for continuously evolving open quantum systems. In a regime of strong coupling, the evolution of such systems is conditioned upon the "measurement" record, which in turn relates to our actions in the external environment, as in our prior observations of photon antibunching in cavity QED. Here we attempt to address the questions of "how much information" quantum mechanics allows us to extract from the atom-cavity system and of the "consequences" of this knowledge on the dynamics of the system itself. We view the atom-cavity system as providing a very fruitful setting for the exploration of such questions in the arena of quantum information dynamics.

\section{The marriage of cavity QED with cold atoms}

Beyond the context of cavity QED, certainly one of the most exciting areas of research in optical physics in recent years has been that of laser cooling and trapping. However, from the perspective of the current discussion, these investigations have been carried out in a regime of weak coupling. In broad conceptual terms, we are attempting to go beyond this state of affairs and to explore the consequences of the marriage of the mechanical motion of atoms with a quantized light field. The central theme of our research is an exploration of the progeny which spring from the union of atom optics with cavity QED, especially with regard to the mechanical consequences of strong coupling. We are interested in situations for which the coupling energy $\hbar g_{0}$ is larger than the atomic kinetic energy $E_{\mathrm{k}}$ and in the interplay of external-state dynamics (associated with atomic center-ofmass motion) with the internal degrees of freedom (for the atomic dipole + quantized cavity field). In the regime $\hbar g_{0}>$ $\left(E_{\mathrm{k}}, \hbar(\gamma, \kappa)\right)$, a single quantum is sufficient to affect profoundly the atomic center-of-mass (CM) motion as well as to excite an appreciable nonlinear response. The experimental requirements for these studies are stringent and have so far been obtained only in our laboratory at Caltech.

From the perspective of continuous quantum measurement for open systems, quantum-state entanglement is generated between fields which escape from the cavity into the external environment (and are then measured) and internal states of the atom-cavity system (including the quantized atomic center-of-mass) [57-60]. Qualitatively distinct evolutions can arise for the atom-cavity system conditioned upon the measurement process (e.g., photon counting vs. heterodyne detection) and its outcome (i.e., the measurement record) in the external environment. Apart from the implicit back-action from the measurement process, explicit feedback can be employed in an attempt to enforce selected coherent evolutions.

For our experiments which combine laser cooled atoms with cavity $\mathrm{QED}$, the normal-mode splitting $\pm g_{0}$ corresponds to a "temperature" $T_{0}=2 \hbar g_{0} / k_{\mathrm{B}}$ which can be much 


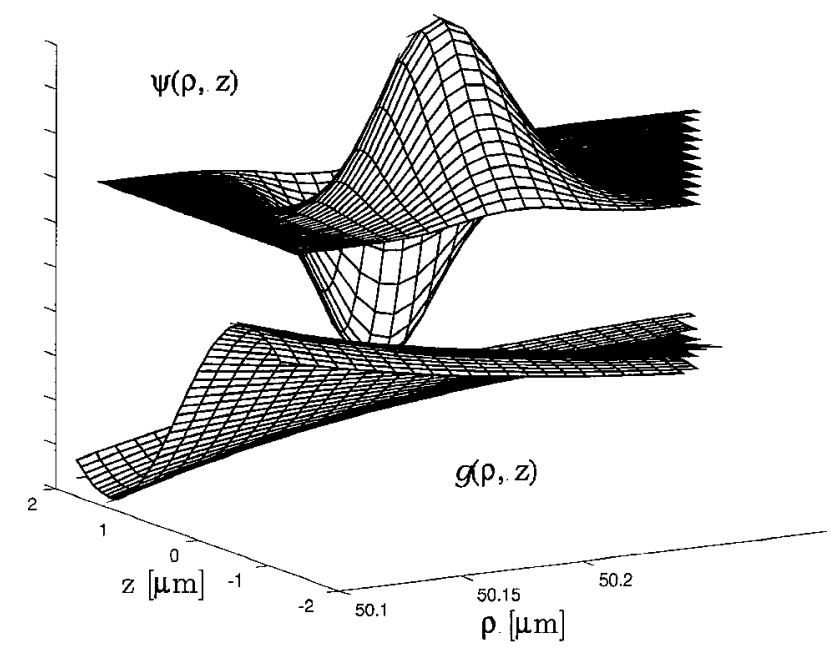

Fig. 5. The center-of-mass wavefunction $\psi(\rho, z)$ and the spatially dependent coupling coefficient $g(\rho, z)$ are seen to have variation on similar spatial scales for a "whispering atom" bound in orbit around a quartz microsphere. Here $\rho$ is the radial coordinate (with the edge of the sphere at $\rho=50 \mu \mathrm{m}$ ), while $z$ measures distance along a line of longitude perpendicular to the equator. The interplay of $\psi(\rho, z)$ and $g(\rho, z)$ gives rise to new structural as well as dynamical phenomena in cavity QED, as analyzed in Ref. $[30,67]$.

larger than the temperatures obtained with polarizationgradient cooling $[61,62]$, with $T \sim 15 \mu \mathrm{K}$ having been achieved in our initial attempts, corresponding to $T_{0} / T \geqslant$ $10^{3}$ for the parameters of eq. (4). In this combined domain of strong coupling with respect to both the internal and external degrees of freedom (i.e., $\left(n_{0}, N_{0}\right)<1$ and $\hbar g_{0} / k_{\mathrm{B}}>E_{\mathrm{k}}$ ) changes in potential energy for motion through a region of spatially varying coupling coefficient $g(\boldsymbol{r})=g_{0} U(\boldsymbol{r})$ (as arises for example in the Gaussian mode $U(\boldsymbol{r})$ of a Fabry-Perot cavity) can profoundly affect the atomic CM dynamics, even for a single intracavity photon $[63,64]$. Likewise, as the atom travels through the cavity with a constant external drive $\mathscr{E}$, the intracavity field $x$ can be substantially modified. Indeed, for weak excitation of the cavity, $x \sim \mathscr{E} / \kappa /[1$ $\left.+2 C_{1}|U(\boldsymbol{r})|^{2}\right]$, where $C_{1}=1 / N_{0}$. For the parameters of eq. (4), this means that the motion of an atom from a node to an antinode can cause a change of the intracavity intensity by a factor $x^{2}>10^{4}$, with a corresponding large modification of the atom's motion. Note that this situation is very different from the usual case for trapped atoms or ions in fixed external potentials, in that here the confining field and the atomic motion are strongly interacting, with the overall state of the system determined in a self-consistent fashion.

Quite recently we have had some success in initial experiments to localize atoms in a cavity with single photons. The basic setup again involves a magneto-optical trap (MOT) situated a few millimeters above a high finesse optical cavity, as shown in Fig. 4. When the MOT is switched off, the atoms fall between the cavity mirrors with some small fraction of the atoms actually transiting through the cavity. With a probe beam tuned to the common atom-cavity resonance, we monitor in real time the transit of an individual atom entering the cavity [34, 48]. Given such a detection event, the next step is to switch the intracavity field to trap the otherwise falling atom. Following the initial calculations of A. S. Parkins and more recent work from the group of D. F. Walls, we make use of the fact that the lower peak of the
vacuum-Rabi splitting corresponds to an attractive (pseudo) potential, with the average "well depth" $\hbar g_{0} \bar{n}$, with $\bar{n}$ as the probability for occupation of the lower dressed state, at least in the limit of weak intracavity fields with photon number $\bar{n} \lesssim 1$. To date, the result of this protocol has been to lengthen the transit time of an individual atom from 100 to beyond $300 \mu \mathrm{s}$, which is to say that the atom spirals within the Gaussian waist for several orbits before being lost. This represents the first demonstration of the role of strong coupling on the mechanical degrees of freedom of an atom in cavity QED and sets the stage for trapping with single photons.

\section{Well-dressed states in cavity QED}

As an atom becomes yet even colder and better localized within the cavity mode, it becomes necessary to consider the full, nonperturbative wave-packet dynamics including bound states for the system. We have thus undertaken an investigation of structure and dynamics for an atom strongly coupled to a cavity mode in the domain for which $E_{\mathrm{k}}<$ $\hbar g$ [30, 67-69]. Beginning with the spectrum of eigenvalues, we have extended the familiar dressed states for the JaynesCummings Hamiltonian [43] to include bound CM states that arise either because of the intrinsic spatial variation of $g(\boldsymbol{r})$ or because of an externally applied atomic potential $V_{\text {ext }}$ $(\boldsymbol{r})$, as for example in an RF Paul trap [70]. Our analysis supplements the system Hamiltonian of eq. (1) with terms for the atomic kinetic energy and an external potential, namely

$H=\frac{\boldsymbol{p}^{2}}{2 m_{\mathrm{a}}}+V_{\mathrm{ext}}(\boldsymbol{r})+\hbar g(\boldsymbol{r}) H_{\mathrm{IF}}$,

where $H_{\mathrm{IF}}=\left(\sigma_{-} a^{\dagger}+a \sigma_{+}\right)$is the Jaynes-Cummings interaction written in a rotating frame at $\omega_{\mathrm{A}}=\omega_{\mathrm{C}}=\omega_{0}$. Here, $\boldsymbol{r}, \boldsymbol{p}$ are $\mathrm{CM}$ position and momentum operators for an atom of mass $m_{\mathrm{a}} \cdot V_{\text {ext }}$ is assumed to have a bound-state structure, and similarly, the term in $g(\boldsymbol{r})$ can be interpreted as an internal state-dependent potential. Spatially localized eigenstates for both the external motion in a potential well and for the internal atom-field interaction are termed "well-dressed" states with the spectrum of eigenvalues which follow from eq. (6) illustrated in Ref. [67].

An emphasis on localized bound states distinguishes our work from most previous treatments of quantized motion in cavity QED [71], which have largely dealt with scattering of unbound momentum eigenstates. By contrast, our analysis explores the interplay of the finite spatial extent of a CM wavepacket $\psi(\boldsymbol{r})$ with the quantum field mode structure $g(\boldsymbol{r})$, as illustrated in Fig. 5. For example, for an atom bound near the surface of a dielectric microsphere, we find significant $(100 \%)$ state-to-state variations in the spontaneous decay rates associated with the overlap of $\psi(\boldsymbol{r})$ and $g(\boldsymbol{r})$. To investigate further the type of dynamical phenomena that can arise, we consider an atom initially localized in the mode $g(\boldsymbol{r})$ with one photon and find substantial modifications to the familiar Rabi nutation rate $2 g(\boldsymbol{r})$ brought about by new CM-dependent spatial and temporal scales implicit in the eigenvalues of the "well-dressed" states. We anticipate issues such as these will become increasingly 
more important as cavity QED moves into a domain with spatially localized atoms.

\section{Evolution of open quantum systems in the presence of feedback}

As intimated in various components of the preceding discussion, we are investigating quantum-limited feedback for the atom-cavity system, following the theoretical lead of $\mathbf{G}$. Milburn and colleagues [72-74]. As noted in [72], the general master equation for homodyne-mediated feedback shares much in common with that describing continuous quantum measurement of position, so that our work in this area is formally as well as practically a natural extension to pursue. Moreover, as applied to studies of atomic CM motion, the setting of cavity QED with a single intracavity atom is unique with respect to the bandwidth and efficiency with which quantum-limited information about atomic motion can be extracted.

With the attainment of the experimental objectives described above, it should be fairly straightforward to implement rudimentary quantum servos, with the first such example being that previously described where a sensing field actuates a trapping potential, with these actions undertaken near the quantum limits for the flow of information to and from the system. To supplement such an operational approach, we are working to develop design principles for the control of experimental systems in a regime of strong coupling, which is a nontrivial undertaking both with respect to implementation as well as empirical assessment. Given the goal of meeting certain specifications for the system density operator $\hat{\rho}$ via quantum-limited feedback, two problems of principle are (1) the estimation of the actual quantum state of the system at any moment and (2) the actuation of a control response conditioned upon this estimate. With regard to estimation, it is clear that the optimal strategy is not necessarily that of inference based upon mean values (e.g., $\langle\boldsymbol{r}\rangle$ from $\langle g(\boldsymbol{r})\rangle$ ), but rather should be derived from more general statistical properties of the detected signal field (e.g., from exclusive probability densities as in the work of Mabuchi in Ref. [56]). Likewise, the criteria for formulating the control law have not been fully developed, but presumably some guidance can be found in the literature on quantum control in molecules [75]. $\mathrm{H}$. Mabuchi is leading this effort in our group.

\section{The one-and-the-same atom laser}

As previously stressed, far and away the most common situation in optical physics is that for which $\left(n_{0}, N_{0}\right) \gg 1$ (weak coupling). Stated somewhat more specifically, for laser operation we have that the threshold for lasing is characterized by a cooperativity parameter $D=M / M_{0} \equiv M D_{1}$, where $M$ specifies the atomic inversion (in number of atoms) and $D_{1}$ is the single-atom cooperativity parameter for the laser [76]. $M_{0}$ is defined in a fashion analogous to $N_{0}$, but now $M_{0}$ depends upon the particular details of the level scheme and pumping process. For conventional lasers, $\left(M_{0}, n_{0}\right) \gg 1$, and consequently a large number of atoms and photons are associated with the lasing threshold, which occurs for $D \simeq 1$. By contrast, note that in our experiments as in eq. (4), we have already achieved a single-atom cooperativity parameter $C_{1} \equiv 1 / N_{0}=80$. The connection between $C_{1}$ and $D_{1}$ is roughly $D_{1} \sim f C_{1}$, where $f$ is the fraction of the total population that gives rise to inversion on the lasing transition. In various circumstances in Cesium, it is possible to achieve $f \gtrsim 0.1$ for candidate upper state lasing transitions and hence to have $D_{1} \gtrsim 8$. Thus it seems reasonable to project "lasing" for $N \sim 1$ atom and much less than one photon, which is a projection substantiated in general terms by the work of $\mathrm{Mu}$ and Savage for one-atom lasers [77]. Much more relevant to the current discussion is the recent analysis by Meyer et al. for the single-ion laser [78].

Within the context of other experimental work in cavity QED to achieve single-atom "lasing", there are two experiments in the microwave domain $[37,38]$ and one in optical regime [79] with $g_{0}>(\kappa, \gamma)$. However, in each case the transit times $T$ are such that $T^{-1} \gg(\kappa, \gamma)$, so that although individual atoms in passing through the cavity are each strongly coupled, steady state is reached only as a result of many atomic transits (so that each atom provides only an incremental effect) and with many photons in the cavity at "threshold". Indeed, An and Feld conclude that their "single-atom" laser is well described by the standard semiclassical laser theory (which is obtained in the weakcoupling limit with $N \gg 1$ atoms) [80], although this is certainly not the case for the experiments in the microwave domain due to the larger values of $g_{0} T \simeq \pi$. In our work we have achieved conditions for which $g_{0}>\gamma>\kappa \gg T^{-1}$ (for the cold atom experiments, $g_{0} T \simeq 10^{3}-10^{4} \pi$ ), so that individual atoms in single transits are responsible for the observed characteristics. Hence, as opposed to previous work in this area with single atoms in the cavity at any one time but with many atoms responsible for the steady-state response [37, 38, 79], our experiments would operate with "one-and-the-same" atom and with a "threshold" photon number $\ll 1$.

\section{Technical advances to the future}

Over the years, our scientific investigations have been enabled by an intertwining of technical advances, which have led to, for example, a reduction of $n_{0}$ by a factor of $10^{8}$ since we embarked on our "mad pursuit" in 1979. In this section, we offer examples of technical engines that might continue to power this enterprize into the future.

To achieve yet higher finesse cavities with increased electric field per photon (i.e., reduced $\kappa$ and increased $g_{0}$ ), we are exploring the whispering gallery modes of small fused-silica spheres (diameter $\sim 100 \mu \mathrm{m}$ ) $[30,81,82]$, as in the pioneering work at Moscow State University [83]. Although there are a number of complex issues related to mode identification and coupling, a central question relates to the quality factors $Q$ that can be attained with these resonators. Projected values range to $Q \simeq 10^{11}$ [83] (which would correspond to a cavity storage time of $50 \mu \mathrm{s}$, with $g_{0} / \kappa \sim 10^{4}$ [81]). In our group, we have recently achieved $Q \simeq 0.8 \times 10^{10}$ at three wavelengths extending into the near infrared (namely, $\{670,780,850\} \mathrm{nm}$ ), with finesse values of $2.2 \times 10^{6}$ [36]. Together with the work Ilchenko et al. [84] 
these measurements represent the highest $Q$ values on record. More importantly, by way of atomic force microscopy of the surface of the microsphere and a simple model we have quantified the role of surface scattering as an important loss mechanism [36], and are formulating strategies to remediate the residual surface roughness (on the nanometer scale).

Relative to the discussion of cavity QED combined with cold atoms, alternate avenues for cooling and trapping within the cavity mode involve the use of a far-off-resonance trap (FORT) $[65,66]$. In such a strategy, the functions of cooling and trapping would be essentially classical in character (and would serve as the "actuator" for control of atomic motion) and would be separate from the quantized atom-field interaction for the resonant mode (which would provide the "sensor"). Yet another prospect that we are following is to employ an ion trap for atomic localization in cavity QED, with the trapping potential corresponding to $V_{\text {ext }}$ in eq. (6).

\section{Coherent processing of quantum information}

From a somewhat more global perspective, we are attempting to lay the foundations for quantum information technology by way of advances in cavity QED. Our vision is to pursue two complimentary paradigms which exploit the atom-field interaction. In the first, the photons become the carriers of quantum information (the "qubits"), with interactions between these "flying" qubits mediated by an atom in a cavity, much as in our prior work with a quantumphase gate [46]. In the second approach, the internal states of atoms are employed as the qubits, with interactions proceeding by way of photons in the intracavity field [85]. These two paradigms are not disjoint, but interactive as in the schemes that we have analyzed for the generation of a deterministic bit of single photon pulses [86] and for the synthesis of entangled states such as that described by Greenberger, Horne and Zeilinger [87]. Surely there are many technical hurdles to overcome (such as for atomic localization within the cavity), but as emphasized in the preceding sections, we are beginning to take significant steps down this road.

Some indication that such a journey is worthy of the effort is provided by our research to combine these capabilities to form quantum networks to implement fundamental quantum communication protocols and for distributed quantum computation [88]. The enabling capability for this work is the successful trapping and localization of atoms inside high-finesse optical cavities. As illustrated in Figs 6 and 7 and as discussed in more detail at the Symposium by P. Zoller (see the contribution by J. I. Cirac), multiple atomcavity systems located at spatially separated "nodes" could be interconnected via optical fibers to create a quantum network $(\mathrm{QN})$ whose unique and powerful properties have been anticipated by recent advances in quantum information theory. Indeed, as led by Professor Zoller's group, a complete set of elementary network operations has been proposed and analyzed including local processing of quantum information, transmission of quantum states from one node to another, and the distribution of quantum entanglements [88-91]. I would emphasize that these proto-

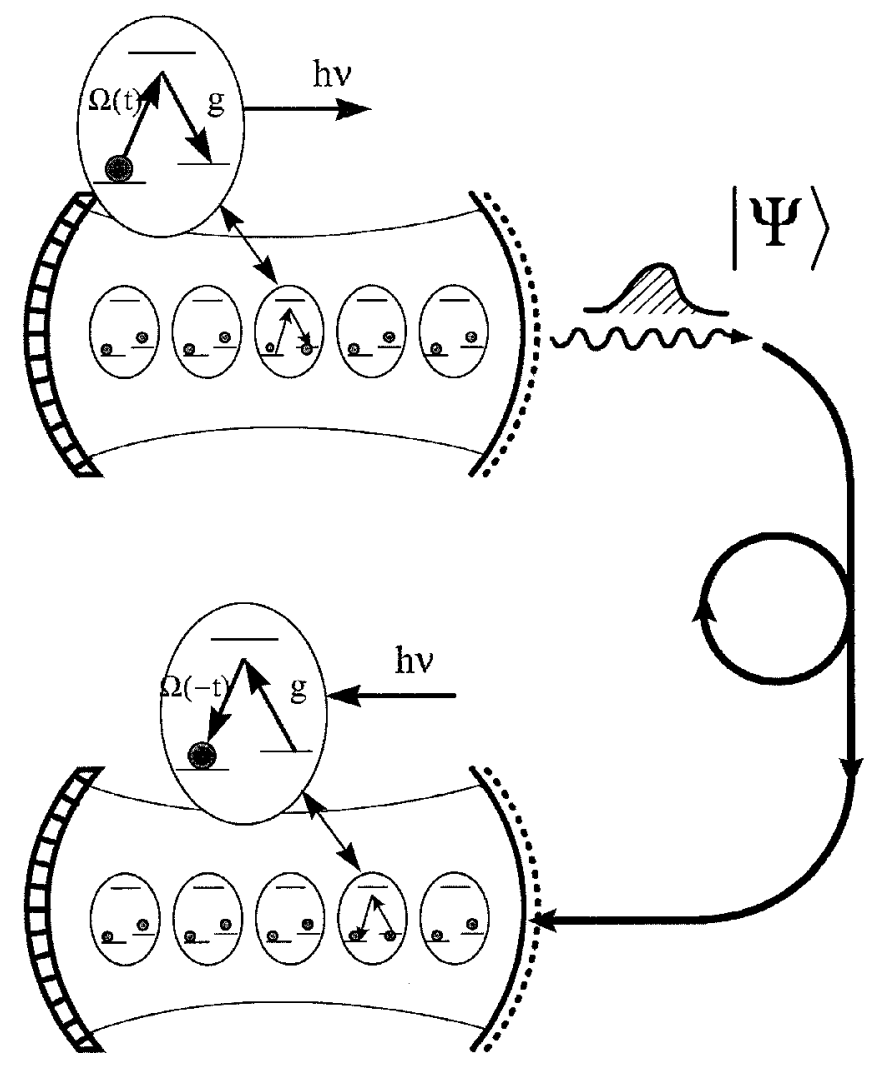

Fig. 6. Illustration of the protocol of Ref. [88] whereby a component of an entangled state for a set of atoms at one site can be transferred to an atom in another set at a remote location. By simple repetition any component of the original state may be so transferred to create nonlocal entanglements.

cols are fully realistic and well within reach of the current technical capabilities, as described in the preceding sections. With respect to quantum computation, we have proposed a distributed paradigm for ultrascale quantum computing that has the potential to overcome size-scaling and errorcorrelation problems through the use of a multiple processor architecture.

\section{Acknowledgements}

This research has been carried out in the Quantum Optics Group at Caltech. The graduate students responsible for the progress described

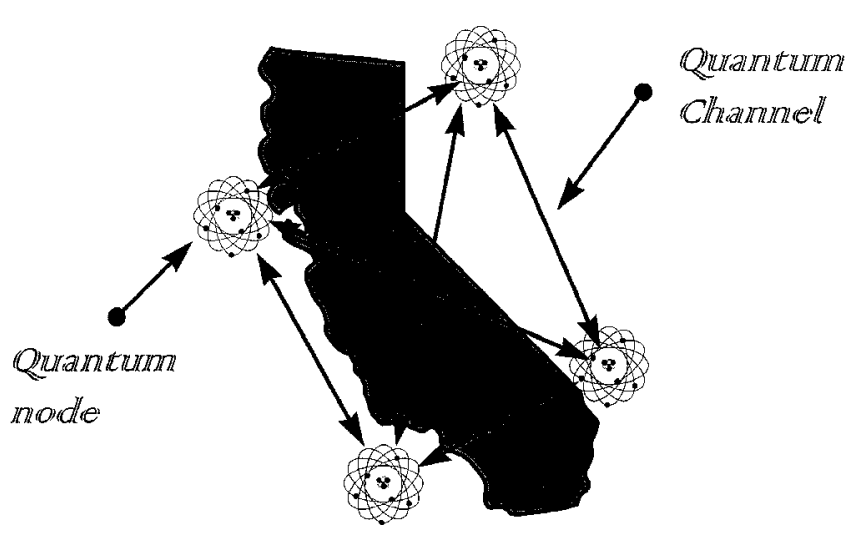

Fig. 7. Schematic of a quantum information network as enabled by capabilities from cavity QED with strong coupling. Internal states of atoms at the quantum nodes are used to generate, process and store quantum information in the fashion of a single cavity in Fig. 6. Photons propagate along the quantum channels to transport quantum states and distribute quantum entanglement following the protocols of Refs [88-91]. 
herein are J. Buck, N. Georgiades, C. Hood, H. Mabuchi, T. Lynn, Q. Turchette and D. Vernooy. Senior members of the group include Drs. M. Chapman, A. Furusawa (Nikon Advanced Research Labs), and W. Lange (now at the MPQ in Garching). We have benefited greatly from ongoing collaborations with the groups of Professors D. F. Walls and P. Zoller.

This work has been supported by the National Science Foundation, by the Office of Naval Research, and by DARPA via the QUIC Institute which is administered by ARO.

\section{References}

1. Einstein, A., Podolsky, B. and Rosen, N., Phys. Rev. 47, 777 (1935).

2. Bell, J. S., "Speakable and Unspeakable in Quantum Mechanics" (Cambridge Univ. Press, Cambridge, 1988).

3. Clauser, J. F. and Shimony, A., Rep. Prog. Phys. 41, 1881 (1978).

4. Braginsky, V. B. and Khalili, F. Ya., "Quantum Measurement" (Cambridge Univ. Press, Cambridge, 1992).

5. Braginsky, V. B., Vorontsov, Y. I. and Thorne, K. S., Science 209, 547 (1980).

6. Thorne, K. S., in "300 Years of Gravitation" (Edited by S. W. Hawking and W. Isreal) (Cambridge University Press, 1987), p. 430, eq. (120).

7. Glauber, R. J., in "Quantum Optics and Electronics" (Edited by C. de Witt, A. Blandin and C. Cohen-Tannoudji) (Gordon and Breach, New York, 1965).

8. Sudarshan, E. C. G., Phys. Rev. Lett. 10, 277 (1963).

9. Kimble, H. J., Dagenais, M. and Mandel, L., Phys. Rev. Lett. 39, 691 (1977).

10. Perhaps the most glaring omission in this condensed history which focuses on the gravitational wave and quantum optics communities is that of the pioneering work of $\mathrm{H}$. Yuen on squeezed states and of Yuen and J. Shapiro on quantum communication. A somewhat more complete overview can be found in Ref. [20] below.

11. Caves, C. M., Phys. Rev. D23, 1963 (1981) and Phys. Rev. D26, 1817 (1982); Caves, C. M. et al., Rev. Mod. Phys. 52, 341 (1980).

12. For a review of research in this era, see "Squeezed States of the Electromagnetic Field," Special Issue of the J. Opt. Soc. Am. B4, 709-1020 (1987).

13. Xiao, M., Wu, L.-A. and Kimble, H. J., Phys. Rev. Lett. 59, 278 (1987).

14. "Quantum Noise Reduction in Optical Systems" Special Issue of Appl. Phys. B55, 189ff (1992).

15. Polzik, E. S., Carri, J. and Kimble, H. J., Phys. Rev. Lett. 68, 3020 (1992).

16. Georgiades, N. Ph., Polzik, E. S., Edamatsu, K. and Kimble, H. J., Phys. Rev. Lett. 75, 3426 (1995)

17. Xiao, M., Wu, L.-A. and Kimble, H. J., Opt. Lett. 13, 476 (1988).

18. Bennett, C. H., Brassard, G. and Ekert, A. K., Scientific American 267, 50 (1992).

19. Ou, Z. Y., Pereira, S. F., Kimble, H. J. and Peng, K. C., Phys. Rev. Lett. 68, 3663 (1992).

20. Kimble, H. J., in "Fundamental Systems in Quantum Optics," Les Houches, Session LIII, 1990, (Edited by J. Dalibard, J. M. Raimond and J. Zinn-Justin) (Elsevier Science Publishers, Amsterdam, 1992), pp. 549-674.

21. Garrett, G. A., Rojo, A. G., Sood, A. K., Whitaker, J. F. and Merlin, R., Science 275, 1638 (1997)

22. Schoelkopf, R. J., Burke, P. J., Kozhevnikov, A. A., Prober, D. J. and Rooks, M. J., Phys. Rev. Lett. 78, 3370 (1997).

23. Ralph, D. C., Black, C. T. and Tinkham, M., Phys. Rev. Lett. 78, 4087 (1997).

24. Steane, A., LANL preprint quant-ph/9708022.

25. Kimble, H. J., in "Cavity Quantum Electrodynamics," (Edited by P. Berman), (Academic Press, San Diego, 1994).

26. Meystre, P., "Progress in Optics," Vol XXX. (Edited by E. Wolf) (Elsevier Science Publishers B. V., Amsterdam, 1982), p. 261.

27. Lugiato, L. A., "Progress in Optics," Vol. XXI, (Edited by E. Wolf) (Elsevier Science Publishers B. V., Amsterdam, 1984), p. 71.

28. Carmichael, H. J., "An Open Systems Approach to Quantum Optics," Lecture Notes in Physics m18 (Springer-Verlag, 1993).

29. Hinds, E. A., in "Advances in Atomic and Molecular Physics" Vol. 20, (Edited by D. Bates and B. Bederson) (Academic, NY, 1985), 347.

30. Vernooy D. W. and Kimble, H. J., Phys. Rev. A55, 1239 (1997).
31. Kimble, H. J., "Highlights in Quantum Optics," in Phil. Trans. R. Soc. Lond. A (1997).

32. Bouwmeester, W. et al., Nature 390, 575 (1997).

33. Braunstein, S. L. and Kimble, H. J., Nature (1998).

34. Hood, C. J., Lynn, T., Chapman, M. and Kimble, H. J., Phys. Rev. Lett. 80, 4157 (1998).

35. Rempe, G., Thompson, R. J., Kimble, H. J. and Lalezari, R., Opt. Lett. 17, 363 (1992).

36. Vernooy, D. W., Ilchenko, V. S., Mabuchi, H., Streed, E. and Kimble, H. J., Opt. Lett. 23, 247 (1998).

37. Brune, M., Raimond, J. M., Goy, P., Davidovich, L. and Haroche, S., Phys. Rev. Lett. 59, 1899 (1987); Bernardot, F., Nussenveig, P., Brune, M., Raimond, J. M. and Haroche, S., Europhys. Lett. 17, 33 (1992).

38. Meschede, D., Walther, H. and Müller, G., Phys. Rev. Lett. 54, 551 (1985); Rempe, G., Klein, N. and Walther, H., Phys. Rev. Lett. 58, 353 (1987); Rempe, G., Schmidt-Kaler, F. and Walther, H., Phys. Rev. Lett. 64, 2783 (1990).

39. Childs, J. J., An, K., Otteson, M. S., Dasari, R. R. and Feld, M. S., Phys. Rev. Lett. 77, 2901 (1996)

40. Thompson, R. J., Rempe, G. and Kimble, H. J., Phys. Rev. Lett. 68, 1132 (1992).

41. Kimble, H. J. et al., in "Proceedings of the International Conference on Atomic Physics 1994," (Edited by D. Wineland, C. Wieman and S. Smith) (American Institute of Physics 1995), 314.

42. Thompson, R. J., Turchette, Q. A., Carnal, O. and Kimble, H. J., Phys. Rev. A (1998).

43. Jaynes, E. T. and Cummings, F. W., Proc. IEEE 51, 89 (1963).

44. Rempe, G., Thompson R. J., Brecha, R. J., Lee, W. D. and Kimble, H. J., Phys. Rev. Lett. 23, 1727 (1991)

45. Turchette, Q. A., Thompson, R. J. and Kimble, H. J., Appl. Phys. B60, S1 (1995).

46. Turchette, Q. A., Hood, C. J., Lange, W., Mabuchi, H. and Kimble, H. J., Phys. Rev. Lett. 75, 4710 (1995).

47. Lange, W., Turchette, Q. A., Hood, C. J., Mabuchi, H. and Kimble, H. J., in "Microcavities and Photonic Bandgaps," (Edited by J. Rarity and C. Weisbuch) (Kluwer, 1996), 443.

48. Mabuchi, H., Turchette, Q. A., Chapman, M. S. and Kimble, H. J., Opt. Lett. 21, 1393 (1996).

49. Wineland, D. J., Itano, W. M. and Bergquist, J. C., Opt. Lett. 12, 389 (1987).

50. Moerner W. E. and Kador, L., Phys. Rev. Lett. 62, 2535 (1989).

51. See the contribution by P. Kwiat. Physica Scripta T76, 115 (1998).

52. Davidovich L. et al., Phys. Rev. Lett. 71, 2360 (1993).

53. Caves, C. M. and Milburn, G. J., Phys. Rev. A36, 5543 (1987).

54. Gagen, M. J., Wiseman, H. M. and Milburn, G. J., Phys. Rev. A48, 132 (1993)

55. Milburn, G. J., Quant. Semiclass. Opt. 8, 269 (1996).

56. Mabuchi, H., Quant. Semiclass. Opt. 8, 1103 (1996).

57. Holland, M. J., Walls, D. F. and Zoller, P., Phys. Rev. Lett. 67, 1716 (1991).

58. Storey, P., Collett, M. and Walls, D. F., Phys. Rev. Lett. 68, 472 (1992) and Phys. Rev. A47, 405 (1993).

59. Averbukh, I. S. et al., Phys. Rev. Lett. 72, 427 (1994).

60. Herkommer, A. M., Carmichael, H. J. and Schleich, W. P., J. Quant. Semiclass. Opt. 8, 189 (1996).

61. Salomon, C., Dalibard, J., Phillips. W. D., Clarion, A. and Guellati, S., E. Phys. Lett. 12, 683 (1990)

62. Monroe, C., Swann, W., Robinson, H. and Wieman, C., Phys. Rev. Lett. 65, 1571 (1990).

63. Haroche, S. Brune, M. and Raimond, J. M., Europhys. Lett. 14, 19 (1991).

64. Englert, B. G., Schwinger, J., Barut, A. O. and Scully, M. O., Europhys. Lett. 14, 25 (1991).

65. Lee, H. J., Adams, C. S., Kasevich, M. and Chu, S., Phys. Rev. Lett. 76, 2658 (1996).

66. Miller, J. D., Cline, R. A. and Heinzen, D. J., Phys. Rev. A47, R4567 (1993).

67. Vernooy, D. W. and Kimble, H. J., Phys. Rev. A56, 4287 (1997)

68. Scully, M. O. et al., Phys. Rev. Lett. 76, 4144 (1996).

69. Ren, W. and Carmichael, H. J., Phys. Rev. A51, 752 (1995).

70. Monroe, C. et al., Phys. Rev. Lett. 75, 4011 (1995).

71. For relevant references, see the citations in Ref. [67] above.

72. Wiseman, H. M. and Milburn, G. J., Phys. Rev. Lett. 70, 548 (1993)

73. Wiseman, H. M. and Milburn, G. J., Phys. Rev. A47, 1652 (1993). 
74. Slosser, J. J. and Milburn, G. J., Phys. Rev. Lett. 75, 418 (1995).

75. Dahleh, M., Pierce, A., Rabitz, H. A. and Ramakrishna, V., Proc. IEEE 84, 7 (1996).

76. Bonifacio, R. and Lugiato, L. A., in "Dissipative Systems in Quantum Optics,” (Edited by R. Bonifacio) (Springer Verlag, Berlin, 1982), p. 2.

77. Mu, Y. and Savage, C. M., Phys. Rev. A46, 5944 (1992).

78. Meyer, G. M., Briegel, H. J. and Walther, H., Europhys. Lett. 37, 317 (1997).

79. An, K., Childs, J. J., Dasari, R. R. and Feld, M. S., Phys. Rev. Lett. 73, 3375 (1994).

80. An, K. W. and Feld, M. S., Phys. Rev. A56, 1662 (1997).

81. Mabuchi, H. and Kimble, H. J., Opt. Lett. 19, 749 (1994).

82. Knight, J. C. et al., Opt. Lett. 20, 1515 (1995) and Opt. Lett. 21, 698 (1996).
83. Braginsky, V. B., Gorodetsky, M. L. and Ichenko, V. S., Phys. Lett. A137, 393 (1989).

84. Gorodetsky, M. L. et al., Opt. Lett. 21, 453 (1995).

85. Pellizzari, T., Gardiner, S., Cirac, J. I. and Zoller, P., Phys. Rev. Lett. 75, 3788 (1995).

86. Law, C. K. and Kimble, H. J., J. Mod. Opt. 44, 2067 (1997).

87. Lange, W. and Kimble, H. J., in preparation (1997).

88. Cirac, J.-I., Zoller, P., Kimble, H. J. and Mabuchi, H., Phys. Rev. Lett. 78, 3221 (1997).

89. Van Enk, S. J., Cirac, J.-I., and Zoller, P., Phys. Rev. Lett. 78, 4293 (1997).

90. Van Enk, S. J., Cirac, J.-I. and Zoller, P., submitted to Science (1997).

91. Van Enk, S. J., Cirac, J.-I. and Zoller, P., quant-ph/9708032 (1997). 